\title{
Asthma Bronchiale and Exercise-Induced Bronchoconstriction
}

\author{
Harshani Jayasinghe Zoe Kopsaftis Kristin Carson \\ Clinical Practice Unit, Respiratory Medicine, The Queen Elizabeth Hospital, Basil Hetzel Institute for Translational \\ Health Research, Woodville South, S.A., Australia
}

\section{Key Words}

Asthma bronchoconstriction - Respiratory illness .

Inflammation

\begin{abstract}
Exercising regularly has a wide range of beneficial health effects; in particular, it has been well documented to help in the management of chronic illnesses including asthma. However, in some individuals, exertion can also trigger an exacerbation of asthmatic episodes and subsequent acute attacks of breathlessness, coughing, tightness of the chest and wheezing. This physiological process is called exerciseinduced bronchoconstriction (EIB) whereby post-exercise forced expiratory volume in $1 \mathrm{~s}$ is reduced by $10-15 \%$ from baseline. While EIB is highly prevalent in asthmatics and presents with similar respiratory symptoms, asthma and EIB are not mutually exclusive. The aim of this review is to present a broad overview of both conditions in order to enhance the understanding of the similarities and differences distinguishing them as two separate entities. The pathophysiology and mechanisms underlying asthma are well described with research now focussing on defining phenotypes for targeted management strategies. Conversely, the mechanistic understanding of EIB remains largely under-described. Diagnostic pathways for both are established and similar, as are pharmacologic and non-pharmacologic treatments and management approaches, which have enhanced success with early detection. Given the potential for exacerbation of asthma, exercise avoidance is common but counterproduc-
\end{abstract}

tive as current evidence indicates that it is well tolerated and improves quality of life. Literature supporting the benefit of exercise for EIB sufferers is at present favourable, yet extremely limited; therefore, future research should be directed in this area as well as towards further developing the understanding of the pathophysiology and mechanisms underpinning both EIB and asthma.

(c) 2015 S. Karger AG, Basel

\section{Introduction}

Regular physical exercise has a plethora of beneficial health effects. Participating regularly in exercise can decrease the incidence of developing heart disease, diabetes and many other health problems. Exercise also helps

Previous articles in this series: 1. Osadnik CR, Rodrigues FMM, Camillo CA, Loeckx M, Janssens W, Dooms C, Troosters T: Principles of rehabilitation and reactivation. Respiration 2015;89:2-11. 2. Holland $A E$, Dowman $L M$, Hill CJ: Principles of rehabilitation and reactivation: interstitial lung disease, sarcoidosis and rheumatoid disease with respiratory involvement. Respiration 2015;89:89-99. 3. Burtin $\mathrm{C}$, Hebestreit $\mathrm{H}$ : Rehabilitation in patients with chronic respiratory disease other than chronic obstructive pulmonary disease: exercise and physical activity interventions in cystic fibrosis and non-cystic fibrosis bronchiectasis. Respiration 2015;89:181-189. 4. Marra AM, Egenlauf B, Bossone E, Eichstaedt C, Grünig E, Ehlken N: Principles of rehabilitation and reactivation: pulmonary hypertension. Respiration 2015;89:265-273. 5. Langer D: Rehabilitation in patients before and after lung transplantation. Respiration 2015;89:353-362.

\section{KARGER 125}

C 2015 S. Karger AG, Basel

0025-7931/15/0896-0505\$39.50/0 
strengthen the immune system, releases 'feel-good' endorphins into the bloodstream and can help in the maintenance of a healthy body weight [1]. Many studies have also shown that physical exercise can help manage and improve symptoms seen in chronic illnesses, one such disease being asthma $[2,3]$. Asthma is a common chronic respiratory illness that can be defined as a chronic but reversible inflammation of the airways. Typically, it is characterised by acute attacks of breathlessness, coughing, muscle tightness in the chest and wheezing [3]. These acute attacks are often caused by asthma triggers such as pollen, common colds or exercising without warming up $[3,4]$.

Exercise-induced bronchoconstriction (EIB) is commonly seen in patients suffering from asthma. It is characterised by wheezing, coughing and chest tightness and is brought on by rigorous exercise [5-8]. In most cases, a decrease in post-exercise forced expiratory volume in $1 \mathrm{~s}$ $\left(\mathrm{FEV}_{1}\right)$ of $10-15 \%$ of the pre-exercise measurement is seen [9-11]. Obstruction of airflow is usually most severe between 3 and 15 min after exercise [12] with a refractory period lasting up to $3 \mathrm{~h}$ following exacerbation of EIB [6].

Due to exercise often being a trigger for asthma, some asthmatics begin to avoid exercise. However, this in fact further deteriorates the poor health of these patients, thus resulting in a deteriorating cycle of exercise avoidance and further deconditioning of the skeletal muscles [13]. Many studies have found that regular exercise can improve asthma symptoms by increasing endurance capacity, lung function and quality of life [3, 14]; however, less is known about the effects of exercise on EIB. Although asthma and EIB are linked, they should be considered separate entities. This review presents a broad overview of asthma bronchiale (more commonly referred to as just asthma) and EIB, in order to distinguish one from the other but also to understand their inherent link. It also aims to provide a summary of diagnosis, treatment and management.

\section{Prevalence}

Asthma is a significant burden for the global community, impacting both quality of life measures and health economics. Given its universal nature, there have been multiple attempts to project worldwide prevalence values over the last decade. Estimates published in 2004 reported that 300 million people were diagnosed with asthma, a figure which is expected to rise by 100 million in the fol- lowing 20 years [15]. This estimate was calculated using data collected between 1988 and 1996 from both the International Study of Asthma and Allergies in Childhood [16] and the European Community Respiratory Health Survey [17], which used inconsistent sampling and recruiting methods and a non-standardised definition for asthma. Furthermore, the reported findings were based upon respondent self-report of wheezing, which, given the heterogeneous nature of asthma, cannot be considered a true indication of clinically diagnosed asthma. The matter of global prevalence was readdressed with emphasis on more robust methods [18]. Data collected from 2002 to 2003 through the World Health Survey [19] indicated that $4.5 \%$ of the world's adult population (18-45 years of age) have been diagnosed with asthma by a doctor or have previously received treatment for asthma (clinical asthma).

While no large-scale global surveys have been undertaken to identify the prevalence of EIB, there have been numerous smaller studies which offer some insight. Approximately, $40-90 \%$ of people suffering from chronic asthma have EIB. EIB is also seen in the non-asthmatic general population, where the prevalence has been estimated to be between 8 and $20 \%$, and is also frequently seen in elite athletes who may or may not have asthma [8].

\section{Pathophysiology and Mechanisms}

Asthma has a complex pathophysiology and a number of underlying mechanisms. Researchers have identified a need to update the definition of asthma, which is presently a description of a collection of non-specific respiratory symptoms that may also be attributed to other pathologies $[5,20]$. During an asthma attack, the muscles in the chest tighten and the airways swell and become inflamed. This inflammation causes a build-up of sticky mucous in the lungs and causes further narrowing of the airways causing breathlessness and the onset of other asthma symptoms [3]. Included in this section is a brief overview of asthma phenotypes, the cellular mechanisms involved and chronic inflammation of the bronchial mucosa occurring in asthma.

Using asthma as an umbrella term, pathophysiological, clinical and demographic characteristics have been clustered together to create phenotypes [20,21]. Numerous studies have highlighted the need to sub-phenotype asthma based on environmental triggers and clinical attributes in order to put in place effective avoidance measures and undertake the correct treatment pathway $[22$, 
Fig. 1. Asthma sub-phenotypes separated by symptom-based, trigger-induced or biomarker-based underlying pathogenesis.

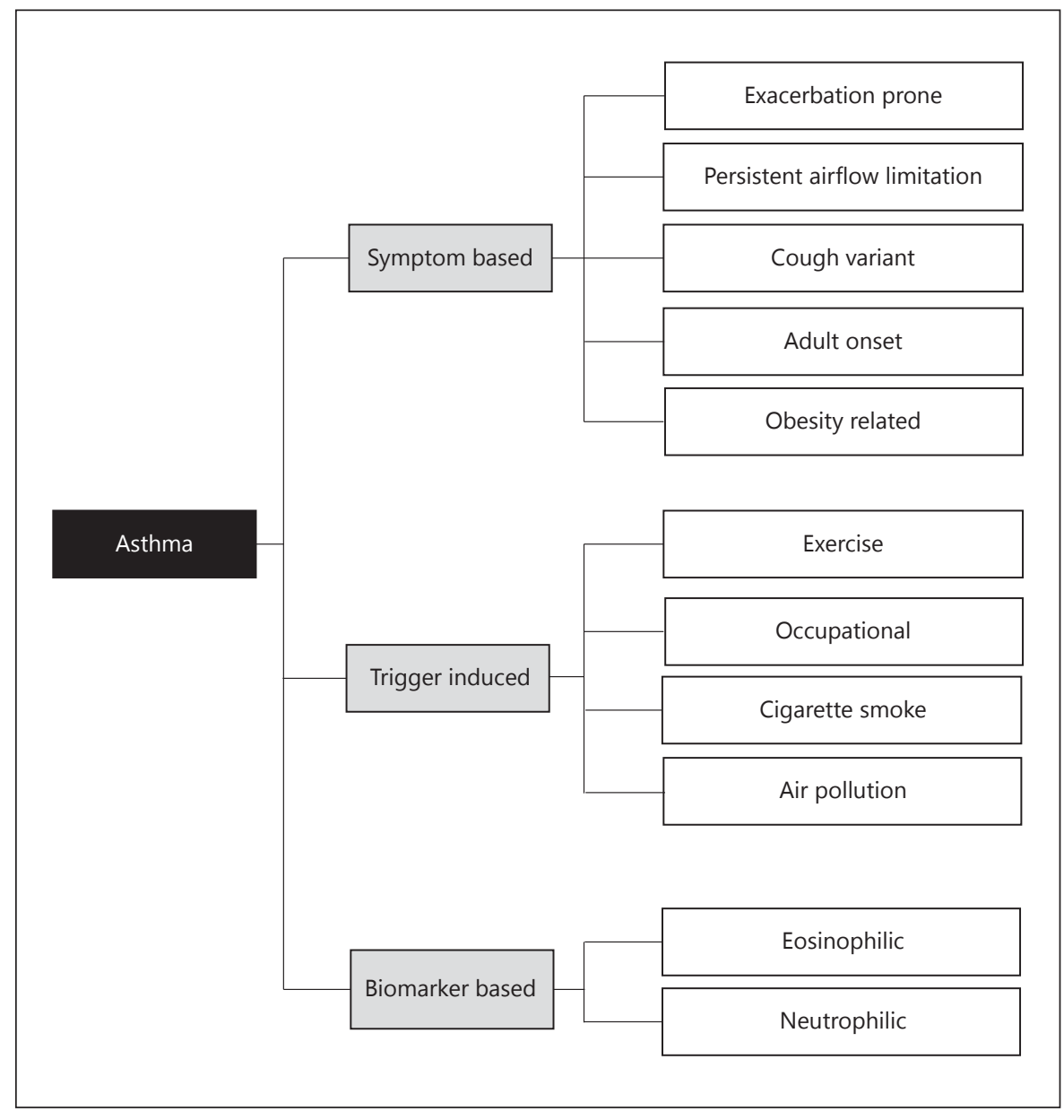

23]. This classification of phenotypes breaks asthma into three major categories: symptom based, trigger induced and biomarker based (fig. 1).

It is possible that with further research both management and treatment may become phenotype specific rather than a blanket approach. Indeed, studies are already using phenotypes as a means of determining treatment for patients, for example the DREAM study, which evaluated 621 severe eosinophilic asthma subjects from 13 countries. This multi-centre double-blind placebocontrolled trial randomised patients to placebo or three varying doses of mepolizumab (from 75 to $750 \mathrm{mg}$ ), finding mepolizumab to be an effective and well-tolerated treatment among this high-inflammation cohort [24]. Yet, despite these targeted treatments, the mechanism by which eosinophilic disease modulates exacerbations remains unknown [25]. More recent reviews acknowledge that airway epithelial muscles are responsible for modulating inflammation and remodelling responses in the airways, yet an understanding of the regulatory mechanisms in epithelial plasticity remains elusive [26,27].

Exploring the individual phenotypes provides the possibility to not only take into account the heterogeneity of asthma but also to begin to understand the physiology and mechanisms underpinning it $[4,7,20,21]$. For example, in allergic asthma, an episode is exacerbated by an allergen [7]. Sensitisation occurs following subversion of the innate immune system through allergens and the associated chemical constituents. Yet, the nature of immune response is still dependent on a variety of external factors including weight (obesity in particular) [28], age of the individual, genetic make-up and timing of exposure, amongst others [29]. In order for the inflammation process to be activated, an allergen is present prompting the release of IgE antibodies (i.e. eosinophils, mast cells and macrophages). The reaction to allergen exposure can be divided into an early phase, characterised by bronchoconstriction occurring within minutes of allergen expo- 
sure, and a late phase, characterised by bronchoconstriction recurring hours later. In early-phase reactions, IgE antibodies secreted by plasma cells bind to mast cells, resulting in the release of mediators, namely histamine and cysteinyl leukotrienes, which act to contract the smooth muscle found in the airways $[30,31]$. The late phase is stimulated by inflammatory cells including monocytes, neutrophils and eosinophils [32] which once again bind to mediators that mediate smooth muscle contraction. Progress has been made in the identification of the basic mechanisms by which allergic disease occurs and the translation of these mechanisms into the human cell systems [29]. Several endogenous anti-inflammatory mechanisms such as lipids and cytokines are also believed to be defective in allergic disease amongst some individuals, thus intensifying inflammation [33]. Importantly, by identifying cellular, genetic and environmental predictors of atopy and the molecular properties of allergens that facilitate sensitisation, these properties can be manipulated for therapeutic purposes [29], though to date developing novel therapies remains a challenge [33].

It has been well established that EIB is triggered by extensive exercise, yet the underlying mechanisms as to why EIB occurs have not been thoroughly investigated. There are, however, several theories as to why it arises. The first suggests that rigorous exercise puts an extra strain on the cardiovascular and respiratory systems causing both cardiac and ventilation output to increase [5]. This intense exercise causes nasal breathing to become substituted by mouth breathing to counter for the extra oxygen needed. A lack of warming of this air by the traditional warming and humidifying process in the nasal passage results in an increase in blood in the lungs and the linings of the bronchial tree, which causes swelling, leading to obstruction and difficulty in breathing [5]. The second theory suggests that EIB is caused by dehydration of the airways as a result of an increase in ventilation. Dehydration is thought to trigger an increase in osmolarity in the airway lining fluid, which in turn causes the release of inflammatory mediators such as histamine, cysteinyl leukotrienes and prostaglandins. These mediators then cause the smooth muscle in the airways to narrow and cause the formation of airway oedema [14].

\section{Athletes and EIB}

An estimated $30-70 \%$ of elite athletes are predicted to have EIB, which can be exhibited in the presence or absence of other asthma characteristics [34]. A study con- ducted among United States of America Olympic winter sports athletes from 1998 found that EIB affected nearly one in every four winter sport athletes, and EIB was found to be more prevalent among women [35]. More recent studies assessing 22 varsity sports have found prevalence estimates for EIB of $39 \%$, with $86 \%$ of EIB-diagnosed subjects (36 of 42 EIB-positive subjects) having no previous knowledge of their positive EIB status [36]. Another 2014 study in Slovenia found similar results among adolescent athletes with $37.5 \%$ being formally diagnosed during the study [37].

Competitive sports depend on an athlete's ability to perform at their peak, as there may be just seconds that separate the first place from the second. It has been suggested that athletes who have EIB may be at a disadvantage compared to those athletes who do not have the condition. However, its negative effect on athletic performance has not yet been proven [34]. During high-level workouts, athletes have to push their bodies to their limits. In doing so, they cause all the systems in the body to work faster and harder. Exercise has been reported to increase ventilation up to $200 \mathrm{l} / \mathrm{min}$ for short periods of time in athletes relying on speed or power and for longer periods in endurance athletes [38]. Due to the extra exertion needed, more oxygen is required to maintain this level of intensity. Simply breathing in through the nose is not efficient enough for the required oxygen; hence, nasal breathing becomes substituted by mouth breathing. One of the results of this exchange is that athletes breathe in a greater amount of not only oxygen but also the surrounding pollutants such as chloramines and ozone and other allergens. Athletes such as swimmers and skiers often train in harsh environments. Swimmers breathe in chlorine regularly and skiers train regularly in harsh cold, dry-air environments. Hence, it is unsurprising that highlevel athletes exhibit a greater amount of respiratory illnesses than the general population, limited not only to asthma [14]. Indeed, a study of winter Olympic athletes found skiers to have the greatest prevalence of EIB among all sports with $57 \%$ of females and $43 \%$ of males diagnosed [35]. Another study on elite summer sports identified a greater risk of asthma among swimmers with 36$79 \%$ showing bronchial hyper-responsiveness to methacholine or histamine; however, fewer studies have investigated the occurrence of EIB [38]. Exposure to these sorts of conditions in the long term can have a significant effect on the airways and can lead to epithelial damage, inflammation and remodelling that often end up resembling patterns seen in asthmatic airways [14]. However, when the strain of intensive exercise is removed, after pe- 


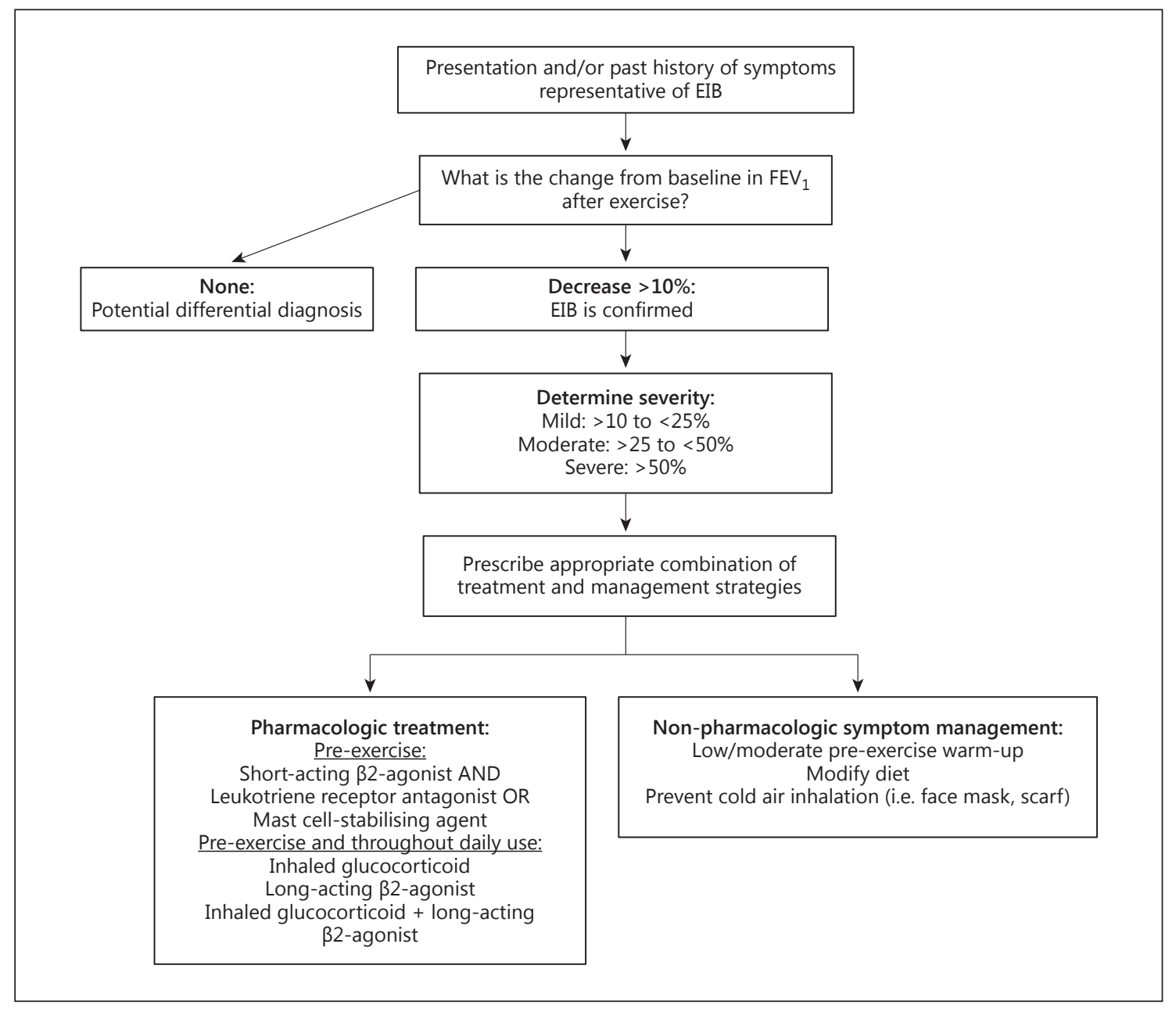

Fig. 2. Diagrammatic representation of diagnosis and treatment options for EIB.

riods of refrainment from these sports, it has been seen that airway hyper-responsiveness can repair and gain normal functioning again, but this can take weeks to years depending on the severity of the damage [14]. Hence, it has been suggested that elite athletes take precautions if possible when training, including not training near pollen-rich fields, wearing protective gear such as masks and avoiding harsh environments for training [14].

\section{Diagnosis}

Diagnosis of asthma is usually done through a lung function test, review of the patient history and also a physical examination. EIB is diagnosed by measuring the change in lung function after exercise. A number of tests can be used to measure lung function including broncho- dilator reversibility testing, which involves the use of a spirometer and a bronchodilator and measures the speed and quantity of air breathed out after exposure to bronchodilators. Bronchoprovocation tests can also be used and are conducted after exposure to asthma triggers such as exercise, mist and cold air $[14,39] . \mathrm{FEV}_{1}$ changes are most commonly looked at after testing; this is mainly due to their strong repeatability as compared to peak expiratory rates. After $30 \mathrm{~min}$ of exercise, a $\mathrm{FEV}_{1}$ percentage is generated, which represents the change in baseline $\mathrm{FEV}_{1}$ minus the lowest $\mathrm{FEV}_{1}$ value generated after exercise. The drop in $\mathrm{FEV} \%$ has to be $>10 \%$ to classify a person as having EIB according to American Thoracic Society (ATS) guidelines. The severity of the condition is classified into mild ( $>10$ to $<25 \%$ ), moderate ( $>25$ to $<50 \%$ ) and severe $(>50 \%)$ [39]. The patient history may reveal a past or current history of wheezing, coughing and tightness of the 
chest resulting from exercise [4]. However, a number of studies have determined that objective confirmation of EIB may occur in the presence or absence of the aforementioned symptoms [29-31] (see fig. 2 for a diagrammatic representation of the diagnosis pathway).

\section{Treatment and Management}

Early detection of asthma and EIB can lead to the development and implementation of a number of strategies that can be employed to reduce the impact that these conditions have on an individual and their quality of life. A combination of non-pharmacological and pharmacological options can be employed for the treatment and management of asthma and EIB (fig. 2). People suffering from asthma are encouraged to see a physician regularly and develop an asthma action plan to prepare for exacerbations. They should also educate themselves and become familiar with the types of activities and environmental stimuli that could trigger their asthma attacks [3]. Lowto-moderate intensity warm-up exercises are recommended before the commencement of intensive exercise for patients that have EIB. This has been shown to reduce EIB in more than $50 \%$ of patients suffering from EIB [14]. Given that inhalation of cold air is associated with exacerbation of EIB, it may be prudent to advise wearing a scarf or something similar immediately prior and during outdoor exercise in order to add moisture and warm the inhaled air. Further non-pharmacologic management strategies include controlling weight, improving fitness and optimising dietary intake [39].

From a pharmacological perspective, the ATS guidelines strongly recommend the use of a short-acting $\beta 2$ agonist for all patients with EIB. Short $\beta 2$-agonists are best used just before exercise (5-10 min prior) and are known to be the most effective drugs against EIB. However, prolonged and frequent use of $\beta 2$-agonists can lead to a tolerance of the drug in the body and a decrease in its protective effects thought to be attributable to a possible down-regulation of the $\beta 2$ receptor [14]. For patients in whom $\beta 2$-agonists are no longer effective, the ATS recommends the use of daily inhaled glucocorticoids such as corticosteroid. Glucocorticoids are not only beneficial for controlling asthma symptoms but can also improve pulmonary function. Furthermore, with regular use, they can also promote a progressive reduction in airway hyperresponsiveness to stimuli like exercise. Long-acting inhaled $\beta 2$-agonists can also be used for treatment and are usually used in conjunction with glucocorticoids when low doses of glucocorticoids are not effective at controlling asthma. However, the ATS does not recommend the daily use of long-acting $\beta 2$-agonists as a single therapy $[14,39]$. The ATS also recommends the use of daily leukotriene receptor antagonists, or a mast cell-stabilising agent, before exercise. It should be noted that there are strong anti-doping bans in many competitive sports, and these medications have restricted use in many sports arenas. It is important for both physician and athlete to be aware of these regulations if an athlete is competing at elite levels [14, 39].

\section{Physical Exercise, Asthma and EIB}

Many people living with asthma and EIB avoid exercise for fear of the onset of symptoms such as wheezing and breathing difficulty. However, it has been found that regular exercise can improve asthma conditions by increasing endurance capacity, lung function and quality of life [14]. Abstinence from exercise worsens asthma symptoms, causes further deconditioning of the airways and results in poorer health outcomes for these individuals. Not participating in exercise also takes a toll on overall quality of life, with many studies showing that lack of engagement in physical exercise, particularly in the early adolescent years, can have a socially isolating impact on individuals $[14,40,41]$. A review published by Carson et al. [3] in 2013 found that exercise was overall well tolerated amongst asthmatic patients and recommended that it should be promoted as no adverse side effects were found in relation to asthma symptoms due to exercise. Although links have been found between exercise and improvements in asthma symptoms, the role that exercise has in improving the symptoms of EIB remains at present more controversial. In a randomised controlled study of children, exposing half of them to exercise training and leaving the other half without treatment, Fanelli et al. [42] found that the children who were exposed to exercise demonstrated a significant reduction in EIB severity as measured by power output and comparison of before and after training values. However, our search managed to find only one study to support this, and other studies looking into this link found inconclusive results.

Further research into the precise mechanisms as to how asthma and EIB occur is needed, which may lead to better treatment and management options. More randomised controlled trials are necessary to evaluate the effects that exercise has on improving EIB, particularly for biological indicators suggesting that exercise may actu- 
ally reduce airway inflammation (for example IgG, histamine responsiveness, sputum and serum eosinophil cell count, C-reactive protein, etc.). Rigorous evaluations examining the effect that EIB has on elite athletes would also be useful in determining if, indeed, EIB has a quantifiable impact on performance.

\section{Conclusion}

The prevalence of asthma and EIB is continuing to rise in developed countries despite improvements in diagnosis and disease management. There are three primary asthma phenotypes, characterised as symptom based, trigger induced and biomarker based. EIB is known to be caused by strenuous exercise, yet the mechanisms underpinning these episodes are still not well known. Subsequently, many people avoid exercise due to fear of an exacerbation or asthma attack, although the current evidence suggests that exercise is not only safe and well tolerated, but can also provide a protective effect through improving exercise endurance and, consequently, the body's ability to cope with strain. Research is needed to gain a better understanding of the mechanisms behind asthma and EIB, which may facilitate improvements in diagnosis, treatment and possibly even prevention.

\section{References}

1 World Health Organization: Global Recommendations on Physical Activity for Health. Geneva, World Health Organization, 2010.

-2 Jankowski M, Niedzielska A, Brzezinski M, Drabik J: Cardiorespiratory fitness in children: a simple screening test for population studies. Pediatr Cardiol 2015;36:27-32.

-3 Carson KV, Chandratilleke MG, Picot J, et al: Physical training for asthma. Cochrane Database Syst Rev 2013;9:CD001116.

4 Global Initiative for Asthma (GINA): Global Strategy for Asthma Management and Prevention. 2014.

5 Park HK, Jung JW, Cho SH, et al: What makes a difference in exercise-induced bronchoconstriction: an 8 year retrospective analysis. PLoS One 2014;9:e87155.

-6 Weiler JM, Anderson SD, Randolph C, et al: Pathogenesis, prevalence, diagnosis, and management of exercise-induced bronchoconstriction: a practice parameter. Ann Allergy Asthma Immunol 2010;105(6 suppl):S1S47.

7 Stevens A, Lowe J, Scott I: Core Pathology, ed 3. Elsevier, 2009.

-8 Krafczyk MA, Asplund CA: Exercise-induced bronchoconstriction: diagnosis and management. Am Fam Physician 2011;84:427-434.

-9 Crapo RO, Casaburi R, Coates AL, et al: Guidelines for methacholine and exercise challenge testing - 1999. This official statement of the American Thoracic Society was adopted by the ATS Board of Directors, July 1999. Am J Respir Crit Care Med 2000;161: 309-329.

10 Rundell KW, Slee JB: Exercise and other indirect challenges to demonstrate asthma or exercise-induced bronchoconstriction in athletes. J Allergy Clin Immunol 2008;122:238246, quiz 247-248.
11 Weiler JM, Bonini S, Coifman R, et al: American Academy of Allergy, Asthma \& Immunology Work Group report: exercise-induced asthma. J Allergy Clin Immunol 2007;119: 1349-1358.

12 Stickland $\mathrm{MK}$, Rowe BH, Spooner $\mathrm{CH}$, et al: Effect of warm-up exercise on exercise-induced bronchoconstriction. Med Sci Sports Exerc 2012;44:383-391.

$\checkmark 13$ Moxham J, Jolley C: Breathlessness, fatigue and the respiratory muscles. Clin Med 2009; 9:778-782.

14 Boulet L, O’Byrne PM: Asthma and exerciseinduced bronchoconstriction in athletes. $\mathrm{N}$ Engl J Med 2015;372:641-648.

15 Masoli M, Fabian D, Holt S, Beasley R; Global Initiative for Asthma (GINA) Program: The global burden of asthma: executive summary of the GINA Dissemination Committee report. Allergy 2004;59:469-478.

16 Beasley R: Worldwide variation in prevalence of symptoms of asthma, allergic rhinoconjunctivitis, and atopic eczema: ISAAC. The International Study of Asthma and Allergies in Childhood (ISAAC) Steering Committee. Lancet 1998;351:1225-1232.

17 Burney P, et al: Variations in the prevalence of respiratory symptoms, self-reported asthma attacks, and use of asthma medication in the European Community Respiratory Health Survey (ECRHS). Eur Respir J 1996;9: 687-695.

18 To T, Stanojevic S, Moores G, et al: Global asthma prevalence in adults: findings from the cross-sectional world health survey. BMC Public Health 2012;12:204.

19 Ustun TB, Chatterji S, Mechbal A, et al: The World Health Surveys; in Murray CJL, Evans DB (eds): Health Systems Performance Assessment: Debates, Methods and Empiricism. Geneva, World Health Organization, 2003, pp 797-808.
20 Wenzel SE: Asthma phenotypes: the evolution from clinical to molecular approaches. Nat Med 2012;18:716-725.

21 Moore WC, Meyers DA, Wenzel SE, et al: Identification of asthma phenotypes using cluster analysis in the Severe Asthma Research Program. Am J Respir Crit Care Med 2010;181:315-323.

22 Hekking PW, Bel EH: Developing and emerging clinical asthma phenotypes. J Allergy Clin Immunol Pract 2014;2:671-680.

23 McCarty JC, Ferguson BJ: Identifying asthma triggers. Otolaryngol Clin North Am 2014;47: 109-118.

24 Pavord ID, Korn S, Howarth P, et al: Mepolizumab for severe eosinophilic asthma (DREAM): a multicentre, double-blind, placebo-controlled trial. Lancet 2012;380:651659 .

25 Burrowes KS, De Backer J, Smallwood R, et al: Multi-scale computational models of the airways to unravel the pathophysiological mechanisms in asthma and chronic obstructive pulmonary disease (AirPROM). Interface Focus 2013;3:20120057.

26 Hackett TL: Epithelial-mesenchymal transition in the pathophysiology of airway remodelling in asthma. Curr Opin Allergy Clin Immunol 2012;12:53-59.

27 Doeing DC, Solway J: Airway smooth muscle in the pathophysiology and treatment of asthma. J Appl Physiol 2013;114:834-843.

28 Farah CS, Salome CM: Asthma and obesity: a known association but unknown mechanism. Respirology 2012;17:412-421.

29 Boyce JA, Bochner B, Finkelman FD, Rothenberg ME: Advances in mechanisms of asthma, allergy, and immunology in 2011. J Allergy Clin Immunol 2012;129:335-341. 
30 Liu MC, Hubbard WC, Proud D, et al: Immediate and late inflammatory responses to ragweed antigen challenge of the peripheral airways in allergic asthmatics. Cellular, mediator, and permeability changes. Am Rev Respir Dis 1991;144:51-58.

-31 Riccio MM, Proud D: Evidence that enhanced nasal reactivity to bradykinin in patients with symptomatic allergy is mediated by neural reflexes. J Allergy Clin Immunol 1996;97:12521263.

32 Peebles RS Jr, Permutt S, Togias A: Rapid reversibility of the allergen-induced pulmonary late-phase reaction by an intravenous beta2agonist. J Appl Physiol 1998;84:1500-1505.

33 Barnes PJ: Pathophysiology of allergic inflammation. Immunol Rev 2011;242:31-50.
34 Price OJ, Hull JH, Backer V, et al: The impact of exercise-induced bronchoconstriction on athletic performance: a systematic review. Sports Med 2014;44:1749-1761.

35 Wilber RL, Rundell KW, Szmedra L, et al: Incidence of exercise-induced bronchospasm in Olympic winter sport athletes. Med Sci Sports Exerc 2000;32:732-737.

36 Parsons JP, Kaeding C, Phillips G, et al: Prevalence of exercise-induced bronchospasm in a cohort of varsity college athletes. Med Sci Sports Exerc 2007;39:1487-1492.

37 Butenko T, Rodman J, Aldeco M, et al: Epidemiology of exercise-induced bronchoconstriction in adolescent athletes with and without asthma in Slovenia. Eur Respir J 2014; 44(suppl 58):P3518.

38 Helenius I, Haahtela T: Allergy and asthma in elite summer sport athletes. J Allergy Clin Immunol 2000;106:444-452.
39 Parsons JP, Hallstrand TS, Mastronarde JG, et al: An official American Thoracic Society clinical practice guideline: exercise-induced bronchoconstriction. Am J Respir Crit Care Med 2013;187:1016-1027.

40 van Veldhoven NH, Vermeer A, Bogaard JM, et al: Children with asthma and physical exercise: effects of an exercise programme. Clin Rehabil 2001;15:360-370.

41 Silva CS, Torres LA, Rahal A, et al: Comparison of morning and afternoon exercise training for asthmatic children. Braz J Med Biol Res 2006;39:71-78.

42 Fanelli A, Cabral AL, Neder JA, et al: Exercise training on disease control and quality of life in asthmatic children. Med Sci Sports Exerc 2007;39:1474-1480. 